\title{
Abnormal laboratory results
}

\section{Interpreting paediatric biochemistry results}

\author{
Peter Verras and Ronda Greaves, Complex Biochemistry Department, Laboratory Services, \\ The Royal Children's Hospital, Melbourne
}

\begin{abstract}
Summary
When interpreting biochemical test results in paediatric patients, consider a number of issues that are associated with and specific to childhood. These include the age of the patient, which may vary from 14 weeks prematurity to more than 18 years, and their body weight, which may range from $\mathbf{5 0 0}$ grams to more than $100 \mathrm{~kg}$. Body size is also a factor in certain situations and is of special concern in the current epidemic of childhood obesity. Children are not miniature adults, however as they age their biochemistry becomes more like that of adults. Added to these patient factors are the effects of the collection process on the blood sample, the method used to analyse the sample, the source of the reference range quoted with the result, and the interpretation placed on the result by laboratory staff.
\end{abstract}

Key words: obesity, prematurity, puberty.

(Aust Prescr 2005;28:126-9)

\section{Introduction}

Historically, the biochemical testing of children, especially very young children, tended to be the province of dedicated 'paediatric' laboratories located in specialist paediatric hospitals. This was usually because small samples of blood were not sufficient for the methods and equipment used in general pathology laboratories at the time. Nowadays almost all of the testing equipment can be adapted to process low-volume samples, although it must be recognised that some of these instruments are more adaptable than others. Most laboratories can now competently analyse small samples without much effect on workflow and consequent productivity, but this does not guarantee that the information provided is adequate for the needs of the referring clinician. The results must be interpreted with a knowledge of the factors that affect children's biochemistry.

\section{Age-dependent factors}

Age-related variation is the single most important factor impacting on the interpretation of paediatric results (Table 1).
To make matters difficult, chronological age is not a completely reliable guide for interpretation of results, as developmental processes are not uniformly linked to the age of the patient. It is often more useful to consider results in terms of the four stages of childhood development:

- neonate - the first four weeks of life

- infant - four weeks to two years

- child - two years to puberty

- adolescent - puberty to adulthood.

Most laboratories report results with reference intervals which are applied to finite age ranges by computer data systems, sometimes causing large and sudden changes in what is considered to be 'normal' when a patient ages by only a few hours. Prematurity, age of onset of puberty and body mass index (BMI) are additional important factors that are not fully taken into account, but can impact significantly on the interpretation of selected biochemical results.

\section{Prematurity}

Despite advances in perinatal care, preterm birth remains a significant problem affecting as many as $6.3 \%$ of all pregnancies. There are more than 16000 preterm deliveries per year in Australia. ${ }^{1}$ Immature and developing organ systems contribute to considerable differences in the concentrations of hormones, proteins, enzymes, metabolites and therapeutic drugs in comparison with adults. It is important to attempt to correct for gestational age especially in the first six months of life. However, most laboratory information systems do not provide such a correction and often this relevant information is not included in the request for the test.

\section{Puberty}

Biochemically, puberty is characterised by the secretion of gonadal hormones. In females puberty can start as early as eight years and is usually completed by the age of 16 years, whereas in boys it generally commences around 10 to 12 years of age and is generally completed by 18 years of age. Reference ranges for biochemical tests are based on chronological age and not on Tanner staging and so results should always be interpreted carefully in this group. There are marked changes in the concentrations of gonadotrophins and steroid hormones during puberty. 


\section{Table 1}

Examples of common analytes where age-related reference ranges need to be considered for the correct interpretation of paediatric results

\begin{tabular}{|c|c|}
\hline Analyte & Reference ranges \\
\hline Albumin & Lower in children, rising from lowest levels in the neonate and infant, to adult levels in adolescence \\
\hline Alkaline phosphatase & Higher in neonates and until post-puberty \\
\hline Bilirubin & Higher in neonates due to immature metabolic pathways \\
\hline Calcium & $\begin{array}{l}\text { Lower values are seen in neonates, especially in prematurity. The upper reference limit is higher in } \\
\text { paediatric patients. Adult ranges apply at about one year. }\end{array}$ \\
\hline Cortisol & No diurnal variation in neonates \\
\hline Creatinine & Lower due to lower muscle mass in children. Adult ranges apply in late adolescence. \\
\hline $\begin{array}{l}\text { Drug concentrations - } \\
\text { relative to dose }\end{array}$ & $\begin{array}{l}\text { Concentrations may be higher in neonates due to immature metabolic pathways, and lower during } \\
\text { childhood due to increased metabolic rates. Reference ranges are usually as for adults. }\end{array}$ \\
\hline Free triiodothyronine & Higher in paediatric patients. Adult ranges apply from late adolescence. \\
\hline $\begin{array}{l}\text { Follicle stimulating } \\
\text { hormone }\end{array}$ & Change to adult levels at puberty \\
\hline Glucose & Lower in neonates, especially in prematurity. Adult ranges apply at one month. \\
\hline Insulin-like growth factor & Change with age throughout life \\
\hline Luteinising hormone & Change to adult levels at puberty \\
\hline Magnesium & Lower in neonates, especially in prematurity. Adult levels apply by about one year. \\
\hline Oestradiol & Change to adult levels at puberty \\
\hline Steroid hormones & Change with age. Assay interference is possible in premature neonates. \\
\hline Testosterone & Change to adult levels at puberty \\
\hline $\begin{array}{l}\text { Thyroid stimulating } \\
\text { hormone }\end{array}$ & Slightly higher in neonates. Adult levels apply at one month. \\
\hline Urea & Higher in neonates, falling to adult levels during infancy \\
\hline Urine catecholamines & Vary with age until adulthood \\
\hline
\end{tabular}

\section{Pre-analytical factors}

The lack of availability of suitably skilled staff for collecting samples from children may affect the quality of the samples received for testing. Collection problems are encountered mainly in neonates and infants, because collecting capillary blood requires some skill and experience to obtain samples which are likely to yield accurate results for all analytes.

Excessive squeezing of a capillary collection site may result in haemolysis, leading to elevation of intracellular analytes such as potassium, magnesium, phosphate and lactate dehydrogenase. Dilution of the sample with tissue fluid can have varying effects on other test results. The consequential small blood sample volume is also more susceptible to the deleterious effects of light, heat, contamination and evaporation than larger-volume venous collections. A frequently encountered problem in small samples is the loss of carbon dioxide into the remaining air space in the tube, leading to falsely low bicarbonate values. The use of inappropriately large specimen containers will exacerbate this and other problems. These factors occasionally generate aberrant results which can only be clarified by repeat collection and analysis.

Collection of timed urine specimens is also often difficult in neonates and infants, and an unusually low timed urine volume must always be viewed with suspicion. Urine output starts at around 100-300 mL per day in infants (3-10 days), increasing with age to adult volumes after puberty. ${ }^{2}$ 


\section{Analytical factors}

For the paediatric biochemist, hormone assays tend to provide the largest challenge in attempting an appropriate balance between cost, turnaround time, and method quality. This is particularly true for full-term and premature babies and for assays that attempt to cater for both the male and female population. Reference ranges provided by different laboratories may vary considerably.

\section{Assay interference}

Fetal adrenal steroids persist until at least 40 weeks post conception, that is, until at least the equivalent of term, despite early delivery. Interference by fetal steroids is not routinely assessed or accounted for by some assay manufacturers, as the premature neonate may not be their main consideration when developing the assay. The potential presence of fetal adrenal steroids should be taken into account when performing and interpreting steroid hormone assays in children less than six months of age. These steroids may interfere with the routine steroid assays available in most laboratories. ${ }^{3}$ It is possible to mitigate these problems by adapting assay methods, but not all laboratories appear to apply these procedures. Even then, different methods may vary widely in their analytical specificity complicating interpretation even further. When in doubt, hormone assays should be repeated at an age equivalent to or greater than that of a full-term pregnancy, or alternatively the tests may be referred to a specialist paediatric laboratory.

\section{Assay imprecision}

In some instances, the choice of assay may be applicable for one section of the population, but may be less than ideal for another section of the population such as children. This was illustrated by a study examining the reliability of results of testosterone assays in females, who have testosterone concentrations comparable to those seen in childhood. The 10 assays examined were the most common assays used in clinical biochemistry laboratories, but they were found to have poor sensitivity and precision for low concentrations of testosterone. $^{4}$

\section{Assay bias (accuracy)}

Bias is a major issue for any laboratory test, and will determine the relevance of quoted reference ranges. Routine tests such as electrolytes and lipids may be closely comparable between different laboratories. However, many others including steroids, peptide hormones, therapeutic drugs and tumour markers will show potentially misleading variation if performed by different laboratories, which may use different kits with different antibody content and specificity.

\section{Interpretation of the results}

The provision of appropriate reference ranges is crucial to the interpretation of any test result, regardless of the patient group involved. The determination of accurate reference intervals is a considerable burden to any laboratory which is increased by the variations in analyte concentrations frequently encountered in young children.

It is therefore possible that a laboratory which does not have access to a large paediatric patient base may not have the resources to determine paediatric reference intervals applicable to its own specific methods and analytical systems. In these circumstances the laboratory may have to depend upon data supplied by the manufacturer of their testing materials, or perhaps determined by other laboratories. It is possible therefore, that the ranges accompanying results may not always be entirely appropriate. Clinicians are well advised to enquire about the source of the reference range(s) when faced with diagnostic uncertainty. Published guidance on paediatric ranges at different ages is available. ${ }^{5}$

Examples of variations which occur in childhood are:

- In the perinatal period, the reference ranges for glucose, calcium and magnesium are lower, while that of bilirubin is higher, than those of other age groups. In addition, when measuring total calcium concentration, it is essential to correct for albumin in newborns, or preferably measure their ionised calcium.

- In the term neonate, bilirubin and drug metabolism pathways are immature and significant changes in concentrations may occur as these pathways mature during the first few weeks following birth. Urea is higher, but falls to adult levels during infancy. These effects are more pronounced in premature neonates.

- From infancy through childhood, serum creatinine and urinary catecholamine excretion are lower, eventually reaching adult levels during adolescence.

- From infancy to adolescence, alkaline phosphatase and insulin-like growth factor-1 change considerably over time.

- Hepatic drug metabolism increases from neonatal levels during childhood, eventually decreasing to adult levels after puberty. Thus, even weight-adjusted doses required to achieve a therapeutic plasma level may be different from those for adults.

Caution needs to be exercised in the interpretation of hormone results from premature babies, because apparently abnormal hormone levels in premature babies may not be indicative of an underlying pathological process. Hormone assays that have negligible interference, and the availability of age-appropriate reference ranges, are essential for correct and timely interpretation of biochemical results in this age group. More work needs to be conducted by laboratories and manufacturers to develop gestational age-appropriate reference ranges for these analytes. 
The pubertal period presents considerable difficulties when assigning reference ranges, since a child may reach puberty earlier or later than may be anticipated. Results may occasionally be seen significantly outside the reference ranges without any apparent pathology. It may therefore be prudent for laboratories not to quote reference ranges for this patient group, especially if there is automated assignment of ranges. Clinicians could consider encouraging their pathology provider(s) to apply interpretative comments instead of possibly incorrect reference ranges. Provision of adequate clinical information to the laboratory will enhance the value of these comments.

\section{Conclusion}

Interpretation of laboratory results from paediatric patients may be made difficult by a number of factors. Where uncertainty remains, it may be advisable to refer further testing to a laboratory which receives relatively larger numbers of paediatric samples and which should consequently have more data and greater experience at interpreting the results.

\section{References}

1. National Health and Medical Research Council. Clinical practice guidelines: Care around preterm birth. Canberra: NHMRC; 1997.
2. Tietz NW, Burtis CA, Ashwood ER. Tietz textbook of clinical chemistry. 2nd ed. Philadelphia, PA:WB Saunders; 1994. p. 2210.

3. Greaves R, Kanumakala S, Read A, Zacharin M. Genital abnormalities mimicking congenital adrenal hyperplasia in premature infants. J Paediatr Child Health 2004;40:233-6.

4. Taieb J, Mathian B, Millot F, Patricot MC, Mathieu E, Queyrel N, et al. Testosterone measured by 10 immunoassays and by isotope-dilution gas chromatography-mass spectrometry in sera from 116 men, women and children. Clin Chem 2003;49:1381-95.

5. Soldin SJ, Brugnara C, Wong EC, editors. Pediatric reference ranges. 4th ed. Washington DC: American Association for Clinical Chemistry Press; 2003.

Conflict of interest: none declared

\section{Self-test questions}

The following statements are either true or false (answers on page 131)

5. Automated reference ranges for biochemical tests may be inaccurate for paediatric patients.

6. Excessive squeezing of an infant's heel to obtain a sample of capillary blood may increase the reported concentration of potassium.

\section{Book review}

\section{Therapeutic Guidelines: Respiratory. Version 3.} Mellbourne:Therapeutic Guidelines Limited; 2005.

\section{5 pages. Price: \$39, students \$25.30, plus postage*}

\section{Cate Howell, General practitioner, Primary Care Mental Health Unit, Department of General Practice, Adelaide}

The latest Therapeutic Guidelines state that the respiratory diseases are largely unchanged but there have been new approaches to them. Since the last Respiratory edition in 2000, new drugs have been introduced and more effective delivery devices and management approaches have been developed. General practitioners require an ever-increasing knowledge base and access to comprehensive information. The Therapeutic Guidelines series are always extremely useful, and incorporate clear summary tables. This respiratory update is timely and welcome.

Given the high prevalence of asthma in Australia, it is highly relevant to have a thorough summary of asthma diagnosis

\footnotetext{
* Available from www.tg.com.au Phone 0393291566
}

and management. This topic is particularly well covered, including risk factor reduction, asthma severity classification and asthma management plans. General practitioners are commonly involved in managing long-term asthma and guidelines are provided. Clinicians will find the summary of treatment of acute asthma attacks in adults and children a useful reference.

The management of chronic obstructive pulmonary disease is well covered, outlines broad management strategies (such as addressing nutritional factors) and emphasises follow-up. The principles of oxygen therapy are outlined early in the book and in this section. Cough can be a problematic presentation and common underlying causes are discussed. Guidelines on conditions such as cystic fibrosis and pleural conditions are provided, and there is a very useful section on sleep apnoea which is increasingly being recognised.

It is important to refresh knowledge of pulmonary function tests regularly, and the section on these is easy to follow, assisted by diagrams. Fitness to fly and scuba dive are covered, as are respiratory drugs in pregnancy and breastfeeding. I would recommend this book as being vital for clinicians to update knowledge and have as a reference. 